\title{
FACTORIZATION AND RANGE INCLUSION OF ADJOINTABLE OPERATORS ON THE WEIGHTED HILBERT $C^{*}$-MODULES
}

\author{
Chunhong Fu, Mohammad Sal Moslehian, \\ QINGXIANG XU AND Ali ZAMANI
}

\begin{abstract}
The indefinite inner products induced by invertible and self-adjoint weights are introduced for elements in Hilbert $C^{*}$-modules. The solvability of the equation $A X=C$ is considered for Hilbert $C^{*}$-module operators. Some equivalent conditions concerning two aspects of factorization and range inclusion are refined and generalized to the weighted case.
\end{abstract}

Mathematics subject classification (2010): 46L08, 47A05.

Keywords and phrases: Hilbert $C^{*}$-module, factorization, range inclusion, weight, reduced solution.

\section{REFERENCES}

[1] M. L. Arias, G. Corach And M. C. Gonzalez, Partial isometries in semi-Hilbertian spaces, Linear Algebra Appl. 428, 7 (2008), 1460-1475.

[2] T. YA. AZIZOV AND I. S. IoKHVIDOv, Linear operators in spaces with an indefinite metric, Translated from the Russian by E. R. Dawson. Pure and Applied Mathematics, New York, A WileyInterscience Publication. John Wiley and Sons, Ltd., Chichester, 1989.

[3] B. A. BARNES, Majorization, range inclusion, and factorization for bounded linear operators, Proc. Amer. Math. Soc. 133, 1 (2005), 155-162.

[4] R. G. Douglas, On majorization, factorization, and range inclusion of operators on Hilbert spaces, Proc. Amer. Math. Soc. 17 (1966), 413-415.

[5] M. Embry, Factorization of operators on a Banach space, Proc. Amer. Math. Soc. 38 (1973), $587-$ 590.

[6] X. Fang, M. S. Moslehian AND Q. XU, On majorization and range inclusion of operators on Hilbert $C^{*}$-modules, Linear Multilinear Algebra 66 (2018), 2493-2500.

[7] X. FAnG, J. YU AND H. YAO, Solutions to operator equations on Hilbert $C^{*}$-modules, Linear Algebra Appl. 431 (2009), 2142-2153.

[8] L. JАКÓBCZYK, Borchers algebra formulation of an indefinite inner product quantum field theory, J. Math. Phys. 25, 3 (1984), 617-622.

[9] E. C. LANCE, Hilbert $C^{*}$-modules-A toolkit for operator algebraists, Cambridge University Press, Cambridge, 1995.

[10] M. S. Moslehian And M. Dehghani, Operator convexity in Krein spaces, New York J. Math. 20 (2014), 133-144.

[11] M. S. Moslehian, M. Kian And Q. XU, Positivity of $2 \times 2$ block matrices of operators, Banach J. Math. Anal. 13, 3 (2019), 726-743.

[12] L. Rodman, A note on indefinite Douglas' lemma, Operator theory in inner product spaces, 225-229, Oper. Theory Adv. Appl. 175, Birkhäuser, Basel, 2007.

[13] J. L. ŠMUL'JAN, Non-expanding operators in a finite-dimensional space with indefinite metric (Russian), Uspehi Mat. Nauk 18, 6 (114) (1963), 225-230.

[14] A. Strohmaier, On noncommutative and pseudo-Riemannian geometry, J. Geom. Phys. 56, 2 (2006), 175-195.

[15] Q. Xu, Y. Chen And C. Song, Representations for weighted Moore-Penrose inverses of partitioned adjointable operators, Linear Algebra Appl. 438, 1 (2013), 10-30. 
[16] Q. XU AND X. FANG, A note on majorization and range inclusion of adjointable operators on Hilbert $C^{*}$-modules, Linear Algebra Appl. 516 (2017), 118-125. 